\title{
Geographic Variation in End-Stage Renal Disease Incidence and Access to Deceased Donor Kidney Transplantation
}

\author{
A. K. Mathur, ${ }^{a}$, V. B. Ashby ${ }^{b}$, R. L. Sands ${ }^{b}$ \\ and R. A. Wolfe \\ ${ }^{a}$ Department of Surgery, University of Michigan, \\ b Scientific Registry of Transplant Recipients, University of \\ Michigan, Ann Arbor, MI \\ 'Scientific Registry of Transplant Recipients, Arbor \\ Research Collaborative for Health, Ann Arbor, MI \\ * Corresponding author: Amit K. Mathur, \\ amitmath@med.umich.edu \\ Note on sources: The articles in this report are based on \\ the reference tables in the 2009 OPTN/SRTR Annual \\ Report, which are not included in this publication. Many \\ relevant data appear in the figures and tables included \\ here. All of the tables may be found online at: \\ http://www.ustransplant.org.
}

\begin{abstract}
The effect of demand for kidney transplantation, measured by end-stage renal disease (ESRD) incidence, on access to transplantation is unknown. Using data from the U.S. Census Bureau, Centers for Medicare \& Medicaid Services (CMS) and the Organ Procurement and Transplantation Network/Scientific Registry of Transplant Recipients (OPTN/SRTR) from 2000 to 2008, we performed donation service area (DSA) and patientlevel regression analyses to assess the effect of ESRD incidence on access to the kidney waiting list and deceased donor kidney transplantation. In DSAs, ESRD incidence increased with greater density of high ESRD incidence racial groups (African Americans and Native Americans). Wait-list and transplant rates were relatively lower in high ESRD incidence DSAs, but wait-list rates were not drastically affected by ESRD incidence at the patient level. Compared to low ESRD areas, high ESRD areas were associated with lower adjusted transplant rates among all ESRD patients (RR $0.68,95 \% \mathrm{CI}$ 0.66-0.70). Patients living in medium and high ESRD areas had lower transplant rates from the waiting list compared to those in low ESRD areas (medium: RR $0.68,95 \% \mathrm{Cl} 0.66-0.69$; high: RR 0.63, 95\% Cl 0.61-0.65). Geographic variation in access to kidney transplant is in part mediated by local ESRD incidence, which has implications for allocation policy development.
\end{abstract}

Key words: Access to care, geography, kidney transplantation, SRTR

Received 30 September 2009, revised 14 December 2009 and accepted for publication 19 December 2009

\section{Introduction}

End-stage renal disease (ESRD) is an extremely debilitating condition and is associated with significant morbidity and mortality. Hundreds of thousands of people in the United States were receiving treatment for ESRD at the end of 2006, and the incidence and prevalence of the disease continue to grow rapidly (1). For eligible patients, kidney transplantation offers a durable treatment with a significant survival benefit and better quality of life compared to lifetime dialysis dependence $(2,3)$. Increased recognition of the significant benefit of transplantation has led to an expansion in the number of patients waiting for a kidney. At the end of 2007, the number of kidney transplant candidates on the waiting list totaled more than 76000 , having grown by $86 \%$ over the preceding decade (4). With growing demand for kidney transplantation in the United States, the identification of patterns of variation in access to kidney transplantation has attracted significant attention in the literature. Several patient and provider-level factors contribute to this variation including patient demographics (5-7), patient race/ethnicity (8-10), the etiology of ESRD (5), the degree of rurality where patients live $(11,12)$ and even ownership status of a patient's dialysis center (13).

One of the most intriguing, and potentially remediable, sources of variation in access to transplantation is the effect of where ESRD patients live. Disparate access to transplantation based on geography is an international phenomena (14-16), and we have previously identified significant geographic variation in access to kidney transplantation across the United States (17). In that study, we demonstrated that the wait-listing, living donor and deceased donor transplant rates varied substantially across donation service areas (DSAs) and states. Many factors could potentially contribute to geographic variation in access to both the kidney transplant waiting list and successful transplantation, but are not well understood. Figure 1 displays a conceptual model of factors that affect access to kidney transplantation in a given geographic area. The factors that will particularly affect access to transplant include differences in patient populations served, variation in organ supply and differences in organ demand. Tremendous variability in effective organ supply may be related to differences in organ donation and conversion rates, organ discard and other factors that organ procurement 


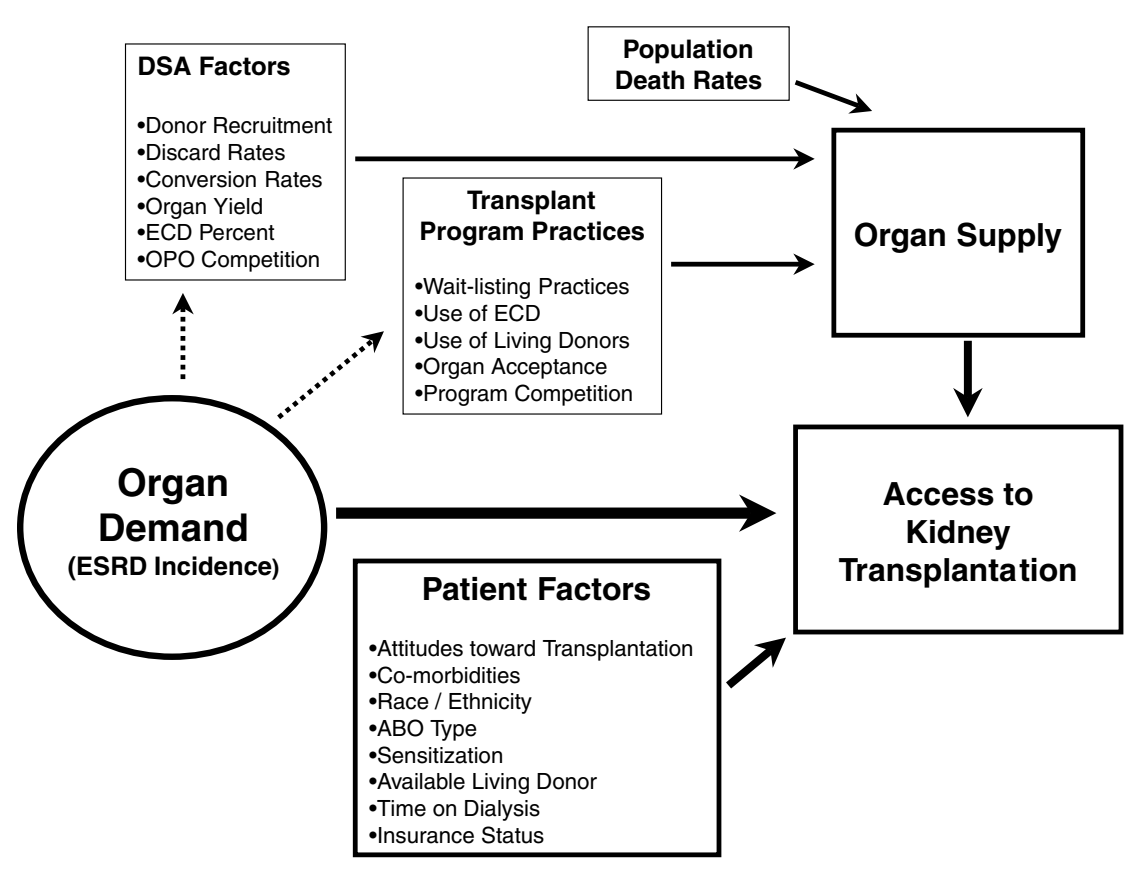

Figure 1: Organ demand affects access to kidney transplant independent of organ supply, provider behaviors and patient characteristics. This conceptual model demonstrates the complex relationship between organ demand, organ supply, patient characteristics and access to kidney transplantation. A patient's individual characteristics including demographics, blood type, antibody status, attitude toward transplantation and other factors are known to affect access to kidney transplantation. Organ demand, our primary variable of interest, is defined as the incidence of ESRD in a given area, and represents a novel mechanism that may affect geographic variation in access to kidney transplantation. The effective organ supply, which can be measured by the overall donation rate in a DSA, also independently affects access to kidney transplantation. As shown, the organ supply is determined by several factors including the population death rate in the area, and the performance and behaviors of OPOs and individual transplant programs. We theorized that organ demand, measured by the incidence of ESRD in a given DSA would independently affect access to kidney transplantation, after accounting for differences in the effective organ supply and patient characteristics.

organizations (OPOs) may affect, and has already led to several policy initiatives (18). We have previously shown that the density of transplant centers in a state or DSA may affect access to kidney transplantation (17), but other center practices related to the use of living donors, ECD organs and organ acceptance protocols may also lead to relative differences in organ supply in different areas. While it has not been directly associated with alterations in donation rates, the density of ESRD in the community, a measure of the demand for organs, may have an effect on organ supply by affecting how transplant programs and OPOs behave. The baseline ESRD incidence and prevalence differs across ESRD geographic networks based on population risk factors, and is growing at dramatically different rates across the country (1), which suggests that geographic variation in demand for kidney transplantation exists. For example, the ESRD rate within a DSA increases with a greater density of African Americans in the population, while the DSA-specific donation rate appears to be insensitive of ESRD incidence (17).

Within this context, we sought to understand how the density of ESRD in the population affects access to the kidney transplant waiting list and successful kidney trans- plantation, after accounting for differences in organ supply. We hypothesized that higher ESRD incidence would be associated with lower wait-list and transplant rates, even after adjusting for the total donation rate. Additionally, we sought to explore how organ supply varies with organ demand within DSAs. In this article, we present the relationship between access to transplant, organ supply and organ demand using DSA-level and patient-level analyses.

\section{Methods}

\section{Data sources}

This article summarizes a special study using data from the U.S. Census Bureau, Centers for Medicare \& Medicaid Services (CMS) and the Organ Procurement and Transplantation Network/Scientific Registry of Transplant Recipients (OPTN/SRTR) from 2000 to 2008. The Population Estimates Program, developed by the U.S. Census Bureau, prepares estimates of the population by age, sex, race and Hispanic origin for the nation, states and counties in the years between censuses (19). The CMS database includes information on all ESRD patients in the United States. The OPTN/SRTR database includes data on all wait-listed kidney transplant candidates, kidney transplant recipients and kidney donors in the United States and is described further in companion articles in this report. The CMS and OPTN/SRTR data sources were supplemented with vital status information 
from the Social Security Death Master File (20). Data from the OPTN Donor Referral database were used to assign the general population and dialysis patients to DSAs (21).

Assignment of DSA was inferred by the county of residence for the general and dialysis populations, and determined by the transplant center of registration for transplant candidates, center where the transplant was performed for recipients, and the location of donation for organ donors. Classifications of race were determined by each data source. Patients were assigned to categories with low or high ESRD incidence. High incidence risk by race was defined as a rate greater than 400 new ESRD patients per million general population. African American and Native American patients were placed in the high incidence category, while whites, Asians and Multiracial/Other race patients were placed in the low incidence category.

\section{DSA-level analyses}

From 2000 to 2008, the count of the general population ranged from 282171936 to 304059724 . Over this period, the total ESRD cases rose to more than 107000 cases per year. There were 243662 waiting list candidates, 83691 kidney transplants and 62622 donors over this period. The average ESRD incidence rate per million population (PMP) for the period was calculated by dividing the sum of the number of ESRD dialysis patients by the sum of the general population and multiplying by one million. Similarly, the average wait-list rate PMP, transplant rate PMP and donor rate PMP were each calculated by dividing the sum of kidney waiting list candidates, kidney transplant recipients and kidney donors, respectively, by the sum of the general population. Wait-list and transplant rates per 100 ESRD population were calculated by dividing the sum of the waiting list and transplant populations, respectively, by the sum of the ESRD population and multiplying by 100 . The transplant rate per 100 waiting list population was similarly calculated by dividing the total transplant population into a denominator comprised of the waiting list population. All rates were also calculated for each DSA and for the low and high incidence populations.

For the DSA-level analyses, we evaluated the individual effects of organ demand (DSA ESRD incidence PMP) and organ supply (DSA-specific donor rate PMP) on three separate metrics of access to kidney transplantation using simple linear regression. Those metrics served as dependent variables in separate models evaluating organ supply and demand and were defined as (1) wait-list rates among the ESRD population, (2) transplant rates among ESRD population and (3) transplant rates among wait-listed candidates. We subsequently created a multivariable regression model to estimate the transplant rate among wait-listed candidates by DSA, using organ supply (DSA-specific donation rate PMP) and organ demand (DSAspecific ESRD incidence PMP) as covariates. This model was additionally stratified by high or low ESRD incidence race. We compared the partial $R^{2}$ for both donation rate and ESRD incidence to determine which contributed the most to the overall model variance.

\section{Patient-level analyses}

The ESRD population was created by assembling records of 662785 ESRD incident patients under the age of 75 , who either began chronic dialysis treatment or were placed on the OPTN kidney or kidney-pancreas waiting list for a first transplant between 2000 and 2008. Patients placed on the kidney waiting list prior to the start of dialysis were considered to have ESRD beginning on the date of wait-listing. Patients who were added to the waiting list on the same date that they underwent a living donor kidney transplant were not counted as having been placed on the waiting list. Patients who had already started dialysis, or were either wait-listed or transplanted prior to 2000, were excluded from the study population. Patients living in a U.S. territory or with an unknown county of residence were also excluded.
To estimate the effects of organ supply and demand on access to kidney transplantation, DSAs were individually categorized into three groups based on kidney donation rates, terciles, and ESRD incidence categories, respectively. Low, medium and high were defined as a rate of less than 21.4 21.4-25.7 and greater than 25.7 PMP, respectively. Similarly, low, medium and high ESRD incidence groups were defined as an ESRD incidence rate less than 300, 300-400 and greater than 400 PMP, respectively. These organ supply and organ demand characteristics for each DSA were subsequently assigned to each patient as described above. We also evaluated several DSA-specific organ supply metrics that may be affected by variations in organ demand in a DSA. These metrics included number of kidney transplant programs, percent of kidney transplants from living donors, percent of transplants from extended criteria donors (ECD), kidney discard rates, donor conversion rates, organ acceptance rates and kidney donor risk index (DRI). These metrics were measured individually for each DSA and grouped into terciles as above. The correlation coefficients were determined from the least squares method.

This study examined how ESRD incidence (low, medium, high) affected access to kidney transplantation for individual patients, adjusted for patient race (low or high ESRD incidence) and donation rates in their DSA (low, medium, high). Separate models were designed to estimate the (1) wait-listing rates among ESRD patients, (2) deceased donor transplant rates among wait-listed patients and (3) deceased donor transplant rates among ESRD patients. These models were designed using multivariable Cox proportional-hazards techniques, and were also adjusted for patient demographics that are captured in the CMS and OPTN/SRTR databases. Patients were followed from the onset of ESRD to the date of wait-listing, from the onset of ESRD to the date of transplantation and from the date of wait-listing to transplantation. The study end-date was December 31, 2008. Follow-up for wait-listing rates and deceased donor transplant rates was censored at death, living donor transplant or end of study. Adjustments for wait-listing rates and deceased donor transplant rates among ESRD patients were patient age, race, ethnicity, sex, cause of ESRD, incidence year (dialysis, wait-listing), comorbid conditions and insurance type. Adjustments for analyses of deceased donor transplant rates among waiting list patients were patient age at wait-listing, race, ethnicity, sex, ESRD cause, wait-listing year, comorbid conditions at wait-listing, insurance type at wait-listing, blood type, panel reactive antibody (PRA) at wait-listing and candidate human leukocyte antigens (HLA). The models provided adjusted relative rates of wait-listing and transplantation, based on the patient's DSA organ supply and demand characteristics. Results are displayed as the relative rates for each level of DSA-specific incidence type compared to the reference rate of 1.00 (low ESRD incidence DSA, low ESRD incidence race, low donation DSA).

\section{Results}

Over the study period, several trends in the ESRD population, kidney transplant waiting list and recipient populations were notable (Figure 2). From 2000 to 2008, the number of patients with ESRD increased by $16.2 \%$, totaling more than 107000 in 2008. Approximately $70 \%$ of ESRD cases were from low incident ESRD races, and nearly $30 \%$ were attributed to the racial groups with high ESRD incidence race. The kidney transplant waiting list grew at a faster rate than the ESRD population, from 21975 to 32722 at the end of 2008 , representing a $48.9 \%$ expansion. In concert with the increases in the ESRD and waiting list populations, the transplant population grew by $30.1 \%$ over the study period, with more than 10000 transplants in 2008 . The population 


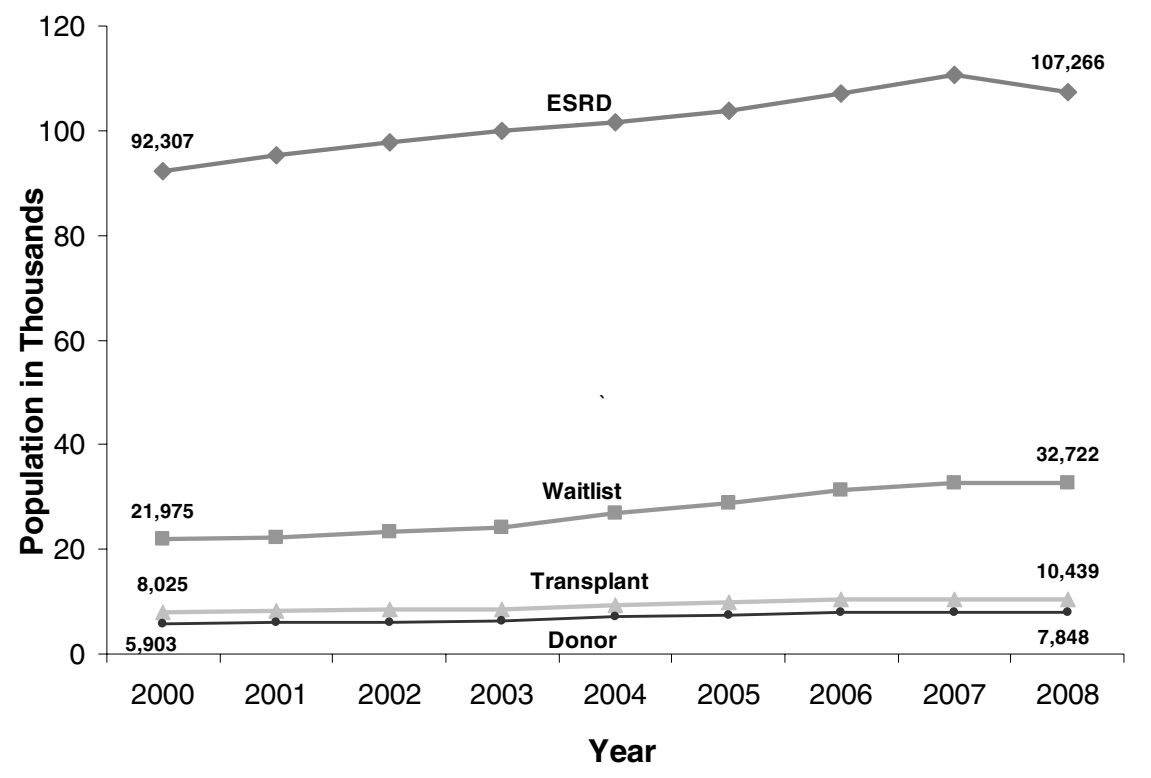

Source: SRTR Special Analysis, August 2009
Figure 2: Growing incident ESRD, new kidney transplant waiting list, kidney transplant and donor populations, 2000-2008. Over an 8-year period, the incidence of ESRD, number of waiting list registrations, kidney transplants and donors has steadily increased. These numbers have increased in each subsequent year, varying by approximately $15-50 \%$ for each metric. of kidney donors grew by $33 \%$ as well, totaling more than 7800 donors at the end of 2008.

Table 1 displays racial differences in the general population, ESRD incidence, kidney transplant wait-listing and transplant rates. The high ESRD incidence group was comprised of $29 \%$ of the total population. Over the study period, 347.1 ESRD cases occurred PMP overall. The high incidence group had 743.4 cases PMP, and the low inci-

Table 1: ESRD, wait-list, transplant and donor rates overall and by ESRD incidence, 2000-2008

\begin{tabular}{|c|c|c|c|}
\hline Population & Total & $\begin{array}{c}\text { High } \\
\text { incidence } \\
\text { race }^{1}\end{array}$ & $\begin{array}{l}\text { Low } \\
\text { incidence } \\
\text { race }\end{array}$ \\
\hline \multicolumn{4}{|l|}{ Counts (n) } \\
\hline General $^{2}$ & 2637315005 & 362339559 & 2274975446 \\
\hline ESRD & 915344 & 269381 & 645963 \\
\hline Wait-list & 243662 & 72952 & 170710 \\
\hline Transplant & 83691 & 26147 & 57544 \\
\hline Donor & 62622 & 9278 & 53344 \\
\hline \multicolumn{4}{|c|}{ Per million general population } \\
\hline ESRD & 347.1 & 743.4 & 283.9 \\
\hline Wait-list & 92.4 & 201.3 & 75.0 \\
\hline Transplant & 31.7 & 72.2 & 25.3 \\
\hline Donor & 23.7 & 25.6 & 23.4 \\
\hline \multicolumn{4}{|c|}{ Per 100 ESRD population } \\
\hline Wait-list & 26.6 & 27.1 & 26.4 \\
\hline Transplant & 9.1 & 9.7 & 8.9 \\
\hline \multicolumn{4}{|c|}{ Per 100 waiting list population } \\
\hline Transplant & 34.3 & 35.8 & 33.7 \\
\hline
\end{tabular}

$\overline{1}$ High incidence races include African Americans and Native Americans.

${ }^{2}$ General population estimates from population division, U.S. Census Bureau (release date: 5/14/2009). dence group had 283.9 PMP. Despite the high prevalence of ESRD in the cohort, there were an average of 92.4 waiting list registrations PMP overall. High incidence groups demonstrated higher wait-list rates versus low incidence groups PMP. Transplant rates averaged PMP were similar between high and low incidence groups (high vs. low incidence: 72.2 vs. 25.3 transplants PMP). Donation rates were low, with an average of 23.7 donations PMP. Amongst the ESRD population, wait-list rates and transplant rates were slightly higher for the high incidence group compared to the low incidence group. However, the high and low incidence groups demonstrated similar transplant rates per 100 waiting list registrations (high vs. low incidence: 35.8 vs. 33.7).

Figure 3 displays the geographic variation in ESRD incidence across the United States by DSA. Fourteen DSAs had less than 300 cases of ESRD PMP, and were classified as low. Thirty-one of the 57 DSAs were of medium ESRD incidence (300-400 ESRD cases PMP), and were primarily found in the eastern parts of the United States and most of California. High ESRD incidence DSAs (>400 ESRD cases PMP) ( $n=12$ ) were concentrated in two geographic areas: parts of the southern and middle Atlantic regions of the county. The geographic variation in DSA-specific kidney donation rates is shown in Figure 4. The distribution of high donation DSAs geographically was highly variable, but the majority were in the eastern United States. The geographic variation in ESRD incidence and donation rate was somewhat discordant. While most high or moderate ESRD areas had simultaneously high or moderate donation rates, two areas demonstrated high ESRD concentration, but low donation rates: the state of Tennessee and northeastern Ohio. Many areas of the country with low ESRD incidence had moderate to high donation rates. 
Figure 3: Geographic variation in ESRD incidence rates by DSA. The majority of the United States geographically has demonstrated a low or medium ESRD incidence over the 8year cohort. High ESRD incidence is concentrated in a few areas of the country, including parts of the southeastern United States, the middle Atlantic region and contiguous areas in the Midwestern United States.

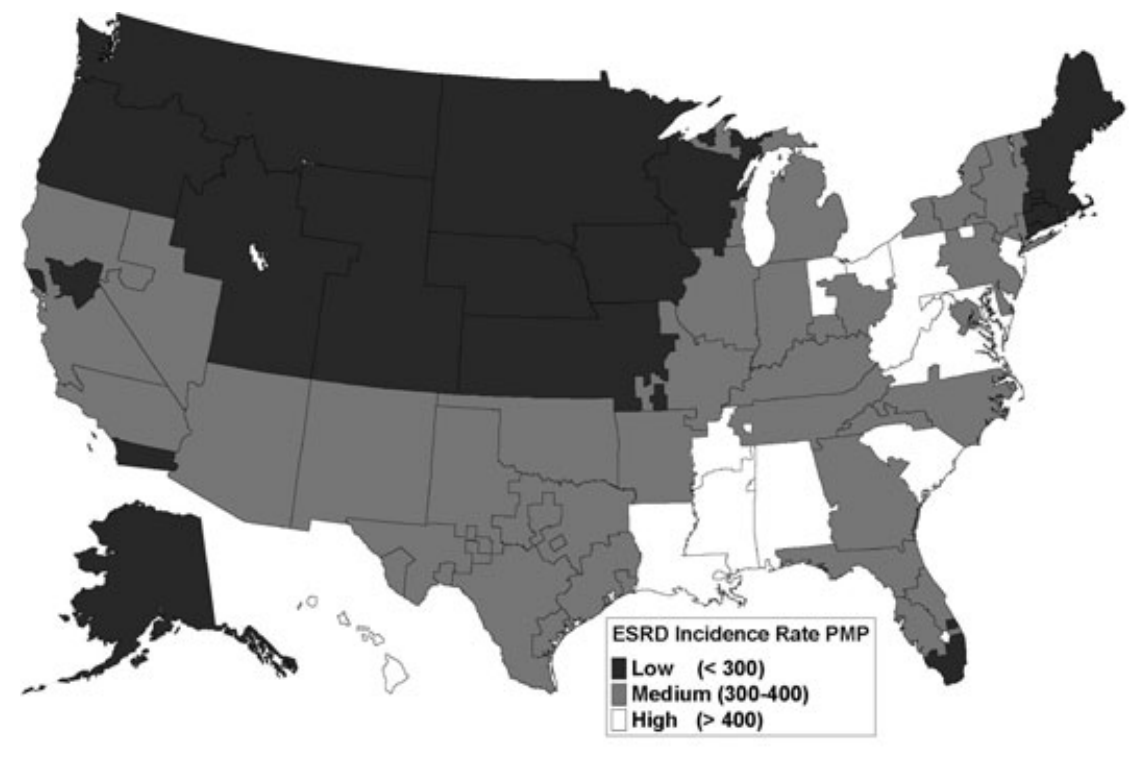

Source: SRTR Special Analysis, August 2009
The six panels in Figure 5 display the complex relationship between access to transplantation, organ supply and organ demand in a DSA-level analysis. Organ supply, as defined by donation incidence PMP, was significantly associated with the transplant rate among 100 ESRD patients. Donation rates were not significantly associated with waitlisting rates or transplant rates from the waiting list. With regards to organ demand, defined as the ESRD incidence PMP, we noted impaired access to kidney transplantation among ESRD patients with increasing concentration of ESRD within a DSA. Wait-list rates among ESRD patients within a DSA declined slightly with increasing incidence, and this trend was also noted in transplant rates from the waiting list. These latter trends were not significant, however. Organ demand and supply significantly affected overall transplant rates from the ESRD population at the DSA level.

Using multivariable regression, we assessed the effect of the donation rate and ESRD incidence within a DSA on its transplant rate among wait-listed candidates for both high and low incidence groups. We found that ESRD incidence within a DSA explained as much of the variance as did the donation rate in predicting transplant rate (partial $R^{2}$ : all
Figure 4: Geographic variation in kidney donation rates by DSA. Compared to the geographic variation seen in ESRD incidence (Figure 3), the United States was more heterogeneous with regards to donation rates. The northeastern and western U.S. states, with the exception of parts of Nevada and contiguous areas, had the lowest donation rates per million population (PMP). Several areas in the southeast, middle Atlantic and Midwest had higher donation rates.

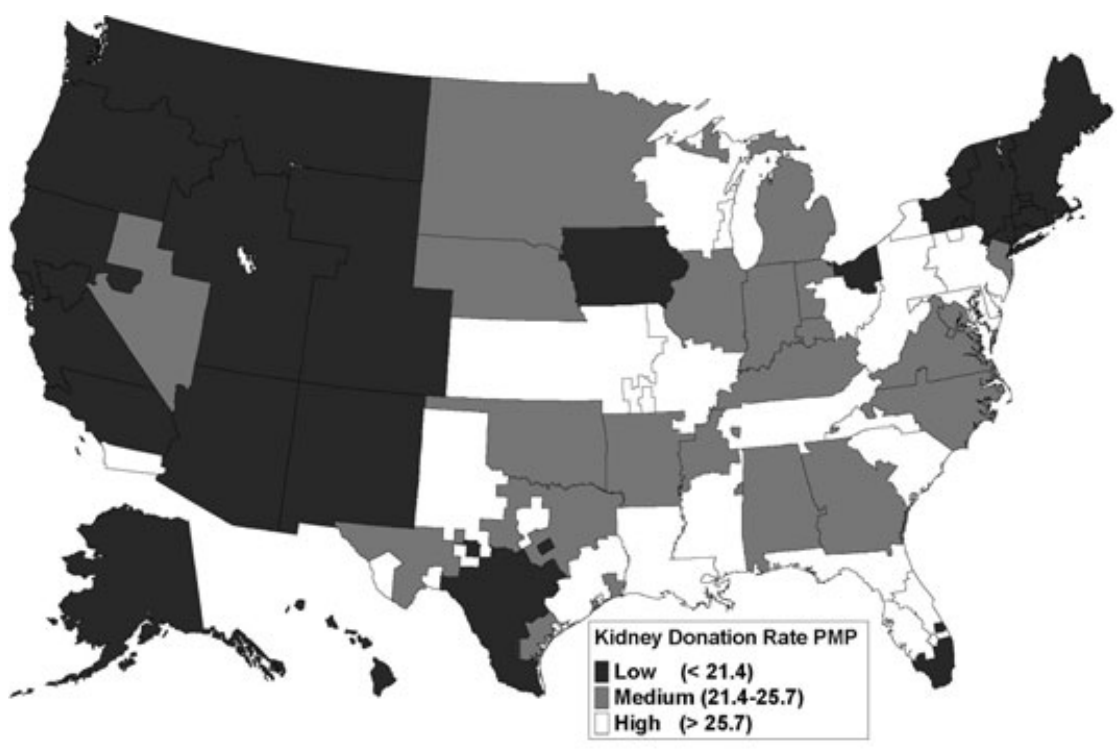

Source: SRTR Special Analysis, August 2009 

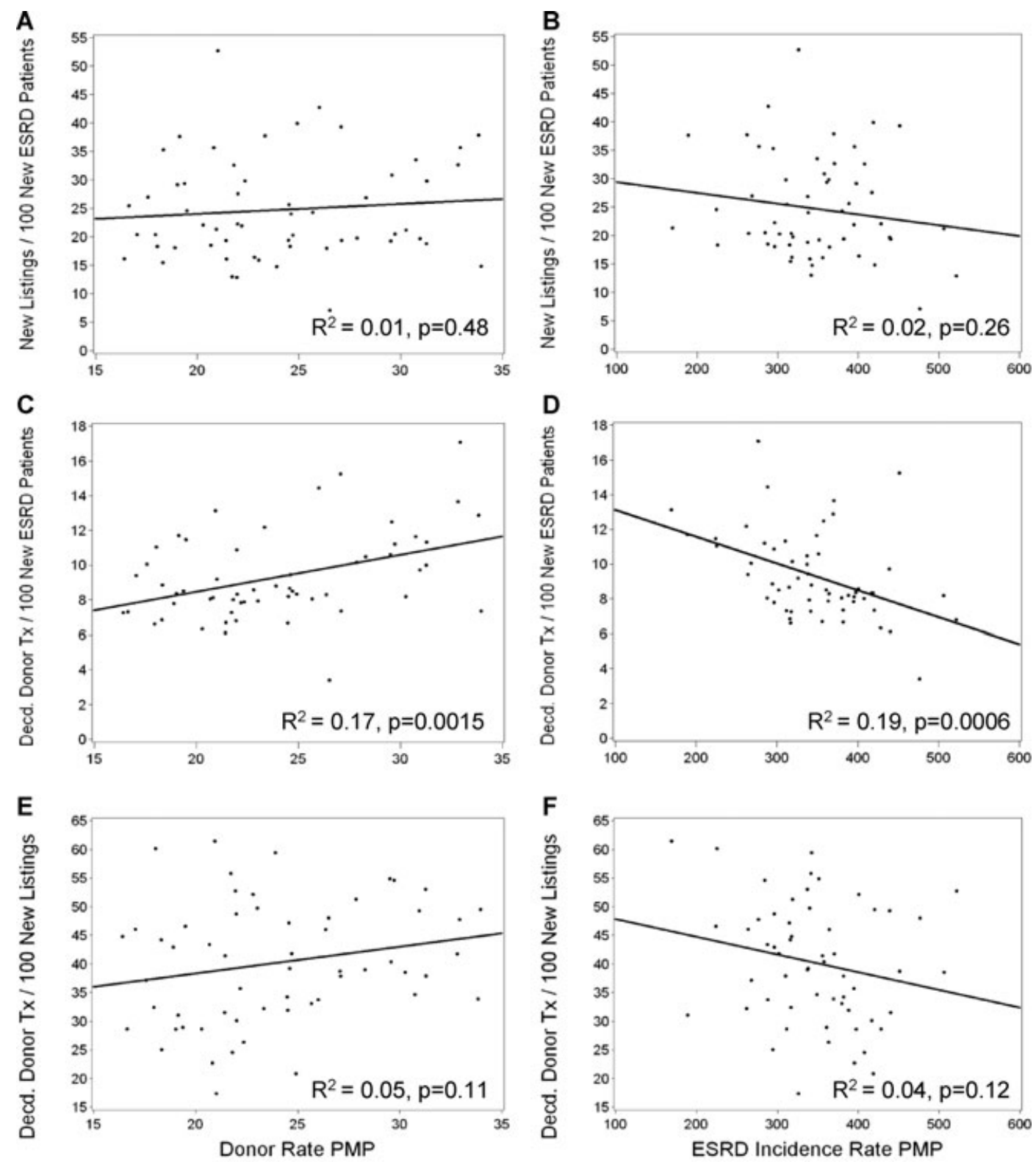

\section{Source: SRTR Special Analysis, August 2009}

Figure 5: Donor organ supply and demand for kidney transplantation. These panels demonstrate six DSA-level models used to evaluate the effect of organ supply (DSA-specific donation rate) and organ demand (DSA-specific ESRD incidence) on three different metrics of access to kidney transplantation: wait-listing rates among ESRD patients $(A, B)$; transplant rate among new ESRD patients (C, D); and transplant rates among wait-listed candidates (E, F). Panel (A) Wait-listing rates among ESRD patients increased slightly, but not significantly, with donation rates. Panel (B) Rates declined with increasing ESRD incidence, but not significantly. Panels (C) and (D) indicate that organ supply was positively correlated with kidney transplant rates among ESRD patients, while increasing ESRD incidence was associated with lower transplant rates. Panels $(E)$ and $(F)$, however, indicate that neither DSA-specific organ supply nor demand was significantly associated with DSA-specific transplant rates from the waiting list.

races: ESRD incidence 0.08 , donation rate 0.08 ) (data not shown). By high incidence or low incidence race in subgroup analyses, ESRD incidence continued to account for a significant proportion of the variance in predictive models for transplant rate within a DSA (partial $R^{2}$ : low incidence race: $E S R D$ incidence, 0.08 ; donation rate, 0.11 ; high incidence race: ESRD incidence, 0.06 ; donation rate 0.08 ) (data not shown). The univariate and multivariate DSA-level 
Variation in ESRD Incidence and Transplant Access

Table 2: Relative rate ${ }^{1}$ of transplant among ESRD patients by subgroup, 2000-2008

\begin{tabular}{|c|c|c|c|c|c|}
\hline Measure & $\mathrm{N}$ & $\%$ & $\mathrm{RR}^{1}$ & p-Value & $95 \% \mathrm{Cl}$ \\
\hline \multicolumn{6}{|c|}{ OPO-donation rate } \\
\hline Low & 226405 & 34 & 1.00 & Ref & \\
\hline Medium & 241950 & 37 & 1.33 & $<.0001$ & $(1.30,1.36)$ \\
\hline High & 194430 & 29 & 1.63 & $<.0001$ & $(1.60,1.67)$ \\
\hline All & 662785 & 100 & & & \\
\hline \multicolumn{6}{|c|}{ Race-ESRD incidence } \\
\hline Low & 438342 & 66 & 1.00 & Ref & \\
\hline High & 224443 & 34 & 0.56 & $<.0001$ & $(0.55,0.58)$ \\
\hline \multicolumn{6}{|c|}{ OPO-ESRD incidence } \\
\hline Low & 104745 & 16 & 1.00 & Ref & \\
\hline Medium & 426746 & 64 & 0.70 & $<.0001$ & $(0.69,0.72)$ \\
\hline High & 131294 & 20 & 0.68 & $<.0001$ & $(0.66,0.70)$ \\
\hline
\end{tabular}

A total of 49627 of 662785 ESRD patients received a primary deceased donor transplant.

Chi-square (Race: 2866, 1 df; ESRD Incidence 909, 2 df; Donation Rate: 1769, 2 df).

${ }^{1}$ Adjusted for patient age, sex, cause of ESRD, incidence year (dialysis, wait-listing), comorbid conditions, employment, BMI and insurance type.

analyses indicated that ESRD incidence had a profound impact on access to kidney transplantation.

We subsequently evaluated the effect of geographic variation in organ demand on access to a primary kidney transplant in a covariate-adjusted patient-level analysis (Table 2). Among the 662785 new ESRD patients, a total of 150193 $(23 \%)$ were placed on the waiting lists for a kidney or kidney-pancreas transplant and 49627 (7\%) received a deceased donor kidney transplant by December 31, 2008. High and moderate donation rates within a DSA led to a 33$63 \%$ higher kidney transplant rate among ESRD patients compared to low donation areas (medium $\mathrm{RR}=1.33,95 \%$ $\mathrm{Cl} 1.30-1.36$; high $\mathrm{RR}=1.63,95 \% \mathrm{Cl} 1.60-1.67)$. High incidence race was associated with a $44 \%$ lower transplant rate versus low incidence race $(R R=0.56,95 \% \mathrm{Cl}$ 0.55-0.58). While simultaneously adjusting for these factors and patient characteristics, increasing ESRD incidence was associated with significantly lower kidney transplant rates. Compared to low ESRD incidence in the patient's DSA, moderate and high ESRD incidence was linked to significantly lower transplant rates, by 30-32\% (medium $\mathrm{RR}=0.70,95 \% \mathrm{Cl} 0.69-0.72$; high $\mathrm{RR}=0.68,95 \%$ $\mathrm{Cl}$ 0.66-0.70). Figure 6 demonstrates the interactions of ESRD incidence, race and donation rate, and the effect on transplant rates among ESRD patients. The effect of ESRD incidence within a DSA was strong; greater incidence was associated with lower transplant rates regardless of donation rates or patient race.

The time-to-waiting list registration model among ESRD patients is displayed in Table 3. Moderate and high donation rates were associated with lower wait-list registration rates (medium $\mathrm{RR}=0.85,95 \% \mathrm{Cl} 0.84-0.86$; high $\mathrm{RR}=0.87,95 \% \mathrm{Cl} 0.85-0.88)$. High ESRD race was associated with a $31 \%$ lower wait-listing rate compared to low ESRD incidence races $(R R=0.69,95 \% \mathrm{Cl} 0.68$ 0.69). Moderate ESRD incidence in the patient's DSA was
Figure 6: Relative rate ${ }^{* *}$ of primary kidney transplant among new ESRD patients by the ESRD incidence rate and donation rate of the patient's DSA, 2000-2008. This figure demonstrates the results of a patient-level analysis evaluating the effect of ESRD incidence, donation rate and race incidence group on access to kidney transplantation among all ESRD patients. Stratified by race incidence group, the effect of medium and high ESRD incidence in the DSA where the patient was transplanted was associated with lower transplant rates, regardless of the patient's race and DSA-specific donation rate.

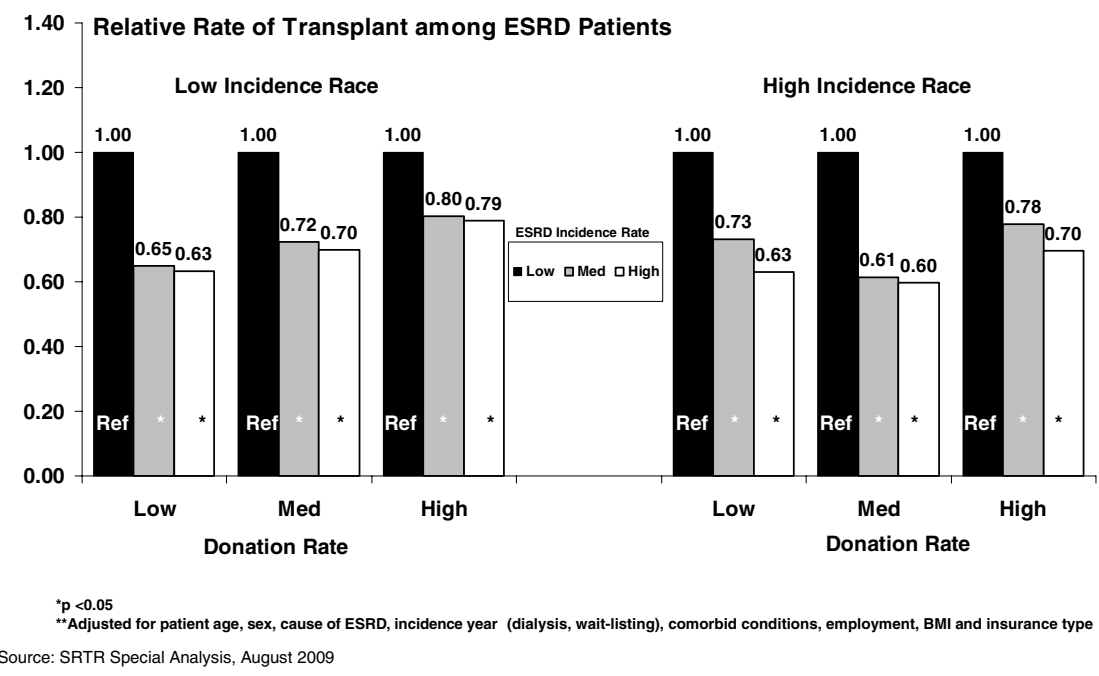


Mathur et al.

Table 3: Relative rate ${ }^{1}$ of wait-listing among ESRD patients, by subgroup, 2000-2008

\begin{tabular}{|c|c|c|c|c|c|}
\hline Measure & $\mathrm{N}$ & $\%$ & $\mathrm{RR}^{1}$ & p-Value & $95 \% \mathrm{Cl}$ \\
\hline \multicolumn{6}{|c|}{ OPO-donation rate } \\
\hline Low & 226405 & 34 & 1.00 & Ref & \\
\hline Medium & 241950 & 37 & 0.85 & $<.0001$ & $(0.84,0.86)$ \\
\hline High & 194430 & 29 & 0.87 & $<.0001$ & $(0.85,0.88)$ \\
\hline All & 662785 & 100 & & & \\
\hline \multicolumn{6}{|c|}{ Race-ESRD incidence } \\
\hline Low & 438342 & 66 & 1.00 & Ref & \\
\hline High & 224443 & 34 & 0.69 & $<.0001$ & $(0.68,0.69)$ \\
\hline \multicolumn{6}{|c|}{ OPO-ESRD incidence } \\
\hline Low & 104745 & 16 & 1.00 & Ref & \\
\hline Medium & 426746 & 64 & 1.02 & 0.002 & $(1.01,1.04)$ \\
\hline High & 131294 & 20 & 1.02 & 0.08 & $(1.00,1.04)$ \\
\hline
\end{tabular}

A total of 150193 of 662785 ESRD patients were placed on the waiting list.

Chi-square (Race: 3939, 1df; ESRD Incidence: 10, 2 df; Donation Rate: 710, 2 df).

${ }^{1}$ Adjusted for patient age, sex, cause of ESRD, incidence year (dialysis, wait-listing), comorbid conditions, employment, BMI and insurance type.

associated with a $2 \%$ higher wait-listing rate compared to low incidence DSAs, but this trend was not significant for high ESRD incidence in the patient's DSA (medium $\mathrm{RR}=1.02,95 \% \mathrm{Cl} 1.01-1.04$; high $\mathrm{RR}=1.02,95 \% \mathrm{Cl}$ 1.00-1.04).

Table 4 displays the results of the time-to-kidney transplant model among wait-listed candidates. Transplant rates increased with increasing donation rates, which was observed in medium and high $158 \%$ and $99 \%$ greater transplant rates [medium $R R=1.58$ and high $R R=1.99$ ], respectively). High incidence race was associated with a $14 \%$ lower transplant rate $(\mathrm{RR}=0.86,95 \% \mathrm{Cl} 0.84-$ 0.88). After adjusting for patient race, donation rate and patient characteristics, ESRD incidence was associated with lower access to kidney transplantation from the waiting list (medium ESRD incidence: $32 \%$ lower transplant rate $[R R=0.68,95 \% \mathrm{Cl} 0.66-0.68]$; high ESRD incidence:
$37 \%$ lower transplant rate $[R R=0.63,95 \% \mathrm{Cl} 0.61-0.65]$; ref $=$ low ESRD incidence $[R R=1.00]$ ).

Finally, the correlation between organ demand and organ supply is displayed in Table 5. The density of kidney transplant programs within a DSA was not associated with ESRD demand $(r=0.03, p=0.85)$. The percentage of kidney transplants from a living donor declined significantly with rising ESRD incidence (46$38 \%$ in low to high incidence tertiles), whereas ECD kidney utilization increased with ESRD incidence (15\% [low]-18\% [high]). DSA-specific kidney discard rates increased with higher organ demand, as did the average kidney DRI. Kidney yield, however, declined with ESRD incidence. Donor conversion and organ acceptance rates did not appear to vary significantly with ESRD incidence $(r=0.08, p=0.57$ and $r=-0.02, p=0.89$, respectively).

Table 4: Relative rate ${ }^{1}$ of transplant among waiting list patients, by subgroup, 2000-2008

\begin{tabular}{|c|c|c|c|c|c|}
\hline Measure & $\mathrm{N}$ & $\%$ & $\mathrm{RR}^{1}$ & $p$ & $95 \% \mathrm{Cl}$ \\
\hline \multicolumn{6}{|c|}{ OPO-donation rate } \\
\hline Low & 58551 & 39 & 1.00 & Ref & \\
\hline Medium & 50946 & 34 & 1.58 & $<.0001$ & $(1.54,1.61)$ \\
\hline High & 40696 & 27 & 1.99 & $<.0001$ & $(1.95,2.04)$ \\
\hline All & 150193 & 100 & & & \\
\hline \multicolumn{6}{|c|}{ Race-ESRD Incidence } \\
\hline Low & 105869 & 70 & 1.00 & Ref & \\
\hline High & 44324 & 30 & 0.86 & $<.0001$ & $(0.84,0.88)$ \\
\hline \multicolumn{6}{|c|}{ OPO - ESRD Incidence } \\
\hline Low & 25647 & 17 & 1.00 & Ref & \\
\hline Medium & 97981 & 65 & 0.68 & $<.0001$ & $(0.66,0.69)$ \\
\hline High & 26565 & 18 & 0.63 & $<.0001$ & $(0.61,0.65)$ \\
\hline
\end{tabular}

A tatal of 49627 of 150193 waitlist patients received a primary deceased donor transplant.

Chi-square (Race: 111, 1df; ESRD Incidence: 1218, 2 df; Donation Rate: 3541, 2 df).

${ }^{1}$ Adjusted for patient age at wait-listing, race, ethnicity, sex, ESRD cause, wait-listing year, comorbid conditions at wait-listing, insurance type at wait-listing, blood type, panel reactive antibody (PRA) at wait-listing, employment, BMI and candidate human leukocyte antigens (HLA). 
Variation in ESRD Incidence and Transplant Access

Table 5: The correlation between organ supply and organ demand in donation service areas, 2000-2008 ${ }^{1}$

\begin{tabular}{|c|c|c|c|c|c|c|}
\hline & \multirow[b]{2}{*}{$\begin{array}{l}\text { United } \\
\text { States }\end{array}$} & \multicolumn{3}{|c|}{ ESRD incidence rate } & \multicolumn{2}{|c|}{ DSA ESRD incidence/PMP } \\
\hline & & $\begin{array}{l}\text { Low } \\
(<300)\end{array}$ & $\begin{array}{l}\text { Medium } \\
(300-400)\end{array}$ & $\begin{array}{l}\text { High } \\
(>400)\end{array}$ & $\begin{array}{l}\text { Correlation } \\
\text { coefficient ( } r \text { ) }\end{array}$ & $\mathrm{p}$-Value \\
\hline Number of donation service areas & 57 & 14 & 31 & 12 & & \\
\hline $\begin{array}{l}\text { Number of transplant centers per } \\
\text { DSA }\end{array}$ & 4.35 & 3.86 & 5.06 & 3.08 & 0.03 & 0.85 \\
\hline $\begin{array}{l}\text { Kidneys transplanted from living } \\
\text { donors (\%) }\end{array}$ & 39.85 & 45.77 & 38.10 & 38.32 & -0.32 & 0.0145 \\
\hline $\begin{array}{l}\text { Kidneys transplanted from } \\
\text { expanded criteria donors (\%) }\end{array}$ & 15.93 & 14.73 & 15.65 & 18.21 & 0.26 & 0.0489 \\
\hline $\begin{array}{l}\text { Kidneys recovered for transplant } \\
\text { but discarded }{ }^{2}(\%)\end{array}$ & 13.87 & 11.68 & 13.73 & 17.06 & 0.41 & 0.0016 \\
\hline $\begin{array}{l}\text { Kidneys per donor }{ }^{3} \text { (number of } \mathrm{KI} \\
\text { txp/number of donors) }\end{array}$ & 1.50 & 1.59 & 1.48 & 1.45 & -0.46 & 0.0004 \\
\hline $\begin{array}{l}2008 \text { Standardized donor } \\
\text { conversion rate ratio }\end{array}$ & 0.99 & 1.05 & 0.96 & 1.02 & -0.06 & 0.63 \\
\hline \multicolumn{7}{|l|}{2008 Organ acceptance rates ${ }^{5}$} \\
\hline Observed rate & 45.7 & 51.6 & 43.8 & 47.3 & 0.08 & 0.57 \\
\hline Expected rate & 45.8 & 44.5 & 46.7 & 43.6 & -0.02 & 0.89 \\
\hline $\begin{array}{l}\text { Average kidney donor risk index } \\
\text { (standard deviation) }\end{array}$ & $1.13(0.44)$ & $1.14(0.41)$ & $1.19(0.43)$ & $1.24(0.46)$ & 0.40 & 0.0022 \\
\hline
\end{tabular}

${ }^{1}$ Source: Special Analysis, November 2009; SRTR data as of July 2009.

${ }^{2}$ Organs recovered for transplant and discarded locally or shared and discarded (i.e. donor disposition $=5$ and donor reason code 503 or 504).

${ }^{3}$ Scientific Registry of Transplant Recipients. Guide to the OPO-specific Reports, July 2009. http://www.ustransplant.org/csr/current/Tech_ notes.aspx. Accessed November 16, 2009.

${ }^{4}$ Ojo AO, Pietroski RE, O'Connor K, McGowan JJ, Dickinson DM. Quantifying organ donation rates by donation service area. Am J Transplant 2005; 5: 958-966.

${ }^{5}$ Wolfe RA, LaPorte FB, Rodgers AM, Roys EC, Fant G, Leichtman AB. Developing organ offer and acceptance measures: when good organs are turned down. Am J Transplant. 2007;7(5 Pt 2):1404-1411.

${ }^{6}$ Rao PS, Schaubel DE, Guidinger MK, Andreoni KA, Wolfe RA, Merion RM, Port FK, Sung RS. A comprehensive risk quantification score for deceased donor kidneys: The kidney donor risk index (KDRI). Transplantation 2009; 88: 231-236.

\section{Discussion}

We hypothesized that geographic variation in access to transplantation, measured at the DSA and patient levels, was a function of organ supply and organ demand. We found that an increasing concentration of organ donors in an area augmented access to kidney transplantation. For patients with ESRD, access to both the waiting list and to transplant after candidate registration was significantly diminished in high ESRD areas compared to low ESRD areas, even after accounting for differences in patient race, other characteristics and donation rates. Increasing disease incidence was associated with diminished access to transplantation at multiple steps in the continuum of care in kidney disease (22). We further demonstrated that organ demand in a DSA is correlated with various organ supply metrics at the DSA level. High ESRD areas were associated with the utilization of a higher proportion of ECD donors, higher kidney discard rates and higher kidney DRI.

The OPTN Final Rule states that barriers in access to transplantation such as geography should be removed in order to provide high quality, equitable care to patients with endstage organ failure (23). Several reports have identified geographic variation in access to kidney and liver trans- plantation $(14,16,17,24-27)$, but these studies primarily described patterns of disparities without providing insight into how this notable variation emerged. Geographic variation has also been identified in earlier steps in the care of patients with chronic kidney disease, such as in patterns of vascular access for dialysis (28). Our study provides a framework in which to consider how geography affects access to care using a plausible mechanism-when scarce resources are allocated, two things determine access: the amount of resources available and the number of people who require those resources. Our analysis represents one of the first efforts to characterize how patterns of geographic variation in the incidence of organ failure affect access to transplant care.

When considered at the DSA or patient level, geography had a substantial effect on access to kidney transplantation, which appeared to be mediated to a significant extent by the incidence of ESRD found in a given area, even after accounting for local donation rates. We evaluated the effect of transplant demand on the system as a whole; the rate of successful kidney transplantation from the total pool of ESRD patients, and then specifically evaluated two steps in the kidney transplant process; access to the waiting list; and access to transplant from the waiting list 


\section{Mathur et al.}

(22). Our findings regarding access to the kidney transplant waiting list raises two important questions related to the size and significance of the effect of the ESRD incidence. First, while the effect of moderate ESRD incidence on wait-list rates was statistically significant, the effect size was small (HR 1.02), and there was no significant effect observed with high ESRD incidence. This phenomenon may be related to how providers make decisions about wait-listing their patients. These decisions are likely driven more by intrinsic patient factors, such as medical criteria, quality of life on dialysis and patient and provider perceptions of transplant risk and survival benefit, as opposed to the epidemiology of ESRD in the surrounding area. The impact of ESRD incidence on wait-list rates, however small, cannot be ignored. High demand for transplant services, measured by the number of patients on dialysis in a given area, may lead to congested waiting lists and longer waiting time. The resources required to provide access to transplant services may be overwhelmed. Transplant providers may not be able to handle the sheer volume of ESRD patients served locally, which may lead to variation in transplant center practices, such as early wait-listing (29). In this context, high ESRD areas may create the perception of super-saturation, creating a sense of urgency for patients on dialysis to seek further medical evaluation required to ultimately improve access to transplant services. The relationship between local ESRD incidence and access to the waiting list is complex, and several unmeasured factors are likely involved in this phenomenon.

The most profound impact of ESRD incidence was on actual transplant rates. Higher ESRD incidence was associated with lower transplant rates among all ESRD patients and the subset on the actual waiting list. Regardless of the denominator, even moderate ESRD incidence was associated with at least a $30 \%$ decrease in kidney transplant rates, and high ESRD incidence was associated with a $32-37 \%$ lower transplant rate. This disparity is of significant clinical concern, because patients who live in relatively ESRD-saturated areas are disadvantaged, and may be precluded from a potential survival benefit with kidney transplantation. The most likely reason for this disparity is related to waiting time. Areas with high ESRD incidence likely contribute to an extended waiting list course, increasing the time candidates must wait on dialysis, which ultimately increases the likelihood of becoming too sick or dying before transplant. The current allocation rules attempt to account for variable waiting time across DSAs by prioritizing time on dialysis rather than waiting time specifically in some areas. If the effect of ESRD incidence is mediated by waiting time, then this policy is substantiated by our findings. Further, the effect of high ESRD incidence may lead to lower transplant rates due to super-saturation of local transplant resources. Patients may not be able to readily work through their diagnostic testing and other transplant waiting list evaluation components, leading to greater inactivation on the waiting list, which makes candidates ineligible for transplant. In recent years, increasing rates of initial Status 7 (inactive status) registrations have emerged (30-33). In high ESRD areas, this may be preferentially done in order to allow patients to accrue waiting time while they finish their diagnostic evaluation, or to accommodate the wait-listing of sicker candidates. High ESRD environments may also be compounded by average to marginal donation rates, which would further decrease the transplant rate. Several mechanisms could potentially mediate the effect of ESRD incidence on transplant rates.

In addition to the independent effect of organ demand on access to transplant, we demonstrated that various organ supply-related factors may vary significantly with ESRD incidence. These relationships further strengthen our conceptual model regarding the effects of organ demand (Figure 1). High ESRD incidence may induce transplant programs and OPOs to optimize potential transplant rates in order to decrease congestion on the waiting list. These mediating effects may be related to use of more ECD organs, resulting in higher than average DRI in high ESRD areas. Living donor transplant rates were negatively associated with ESRD incidence, which may reflect the current state of practice patterns in transplant programs, but also may be related to the potential of less available eligible living donors in high ESRD areas. With the growing knowledge of the benefits of living kidney transplantation and the safety of organ donation, this phenomenon may change, but living donor candidacy may continue to be a problem in ESRD-rich areas. Organ yield and discard rates declined with higher ESRD incidence, which may be related to a greater tendency to procure donor kidneys to increase access to transplantation, but results in the discard of a high proportion of inadequate kidneys. These data indicate that successful kidney transplantation is driven by complex epidemiological phenomena related to the availability of scarce resources, and how transplant organizations respond to these forces in order to provide the best care for their patients.

Our analysis also substantiates numerous analyses regarding racial disparities in access to kidney transplantation by accounting for the effect of geography. We compared racial groups based on the incidence of ESRD in a specific population. The high ESRD incident groups, comprised of African Americans and Native Americans, had relatively less access to the waiting list and transplantation compared to low incidence ESRD racial groups (whites, Asians and those of Other/Mixed race), while adjusting for patientlevel factors. These disparities are likely driven by the differences in access between whites and African Americans, since they made up the respective majorities in each group. African American race has been previously associated with failure to progress through the transplant process (34-37). The racial differences in access have been attributed to several factors, including patient preferences and provider attitudes $(9,38-40)$, and programs have been initiated to increase minority access to transplant (41-43). Our findings 
dovetail with previous studies addressing racial disparities in access to kidney transplantation.

Our evaluation on how geographical variation in ESRD incidence affects access to transplantation has some limitations. This is an observational study based on registry data. Due to the methodological design of this study, causal inferences cannot be made regarding high ESRD incidence and access to transplantation. Unmeasured factors, such as socioeconomic status, that affect access to transplant within a geographic area potentially could confound our findings. We have also considered disease incidence over a 9-year period, and practices that potentially affect access to transplant may have had differential effects within areas of high ESRD incidence. We acknowledge these factors in our conceptual model (Figure 1), and may include the utilization of extended criteria kidneys, desensitization protocols and living donor kidney transplants. Center and provider practices in both high and low ESRD areas may certainly contribute to the patterns in access noted in our study, particularly with regards to competition, which we have established has detrimental effects on relative kidney transplant rates (17). This study also does not account for individual medical decision-making, which accounts for patient preferences and clinical factors not necessarily captured in our data. Despite these limitations, our study represents one of the only attempts to characterize the mechanism of geographic variation in access to transplantation.

The fact that the rate of endemic ESRD influences access to transplantation for two otherwise similar ESRD patients living in different areas has significant policy implications. In the context of the Final Rule, this inequity should potentially be addressed in the policies governing the allocation of kidneys. The current kidney allocation system is under review by the OPTN and the transplant community, and future allocation paradigms should address geographic inequities more broadly. Increased organ sharing with high ESRD areas could have a tremendous impact on improving equity without necessarily diminishing the utility of the donated organ. With a $2 \%$ higher waitlisting rate in high ESRD areas and a 37\% lower transplant rate from the waiting list in these areas compared to low ESRD areas, it is clear that geographic disparities are problematic in a very tangible way, and that policies that include rules to help remove this inequity should be encouraged. We have also demonstrated that high organ supply, in addition to organ demand, is associated with higher wait-listing and transplant rates. The effective organ supply in a given area may be driven by a variety of factors that are determined by OPO and transplant program behavior. Increasing utilization of ECD kidneys, aggressive organ acceptance practices and competition between transplant programs may affect transplant rates. We have previously evaluated how competition affects kidney transplant rates, and have shown that more competition actually resulted in lower, rather than higher, transplant rates (17).
Policies to increase the effective organ supply that focus on transplant program and OPO performance may result in greater access to kidney transplantation, and may overcome the barriers related to geographic variation in organ demand

In summary, high ESRD incidence in a given geographic area is associated with lower access to transplant, regardless of race/ethnicity. Racial/ethnic disparities in access to the kidney waiting list and to transplant for wait-listed candidates were notable, even after accounting for differences in donation rates and ESRD incidence. These findings further elucidate the mechanisms of geographic disparities in access to transplantation, and policy makers should consider these disparities in allocation policy reform.

\section{Acknowledgments}

Disclaimer: The Scientific Registry of Transplant Recipients is funded by contract number 234-2005-37009C from the Health Resources and Services Administration (HRSA), US Department of Health and Human Services. The views expressed herein are those of the authors and not necessarily those of the US Government. This is a US Government-sponsored work. There are no restrictions on its use. This study was approved by HRSA's SRTR project officer. HRSA has determined that this study satisfies the criteria for the IRB exemption described in the 'Public Benefit and Service Program' provisions of 45 CFR 46.101(b)(5) and HRSA Circular 03. This article was edited by Jennifer McCready-Maynes of the Arbor Research Collaborative for Health.

\section{Conflict of Interest Statement}

The authors declare no conflicts of interest.

\section{References}

1. U.S. Renal Data System. USRDS 2008 Annual Data Report: Atlas of Chronic Kidney Disease and End-Stage Renal Disease in the United States. Bethesda, MD: National Institutes of Health, National Institute of Diabetes and Digestive and Kidney Diseases. 2008. Available from: www.usrds.org/atlas.

2. Wolfe RA, Ashby VB, Milford EL et al. Comparison of mortality in all patients on dialysis, patients on dialysis awaiting transplantation, and recipients of a first cadaveric transplant. N Engl J Med 1999; 341: 1725-1730.

3. Liem YS, Bosch JL, Arends LR, Heijenbrok-Kal MH, Hunink MG Quality of life assessed with the Medical Outcomes Study Short Form 36-Item Health Survey of patients on renal replacement therapy: A systematic review and meta-analysis. Value Health 2007; 10: 390-397.

4. McCullough KP, Keith DS, Meyer KH, Stock PG, Brayman $\mathrm{KL}$, Leichtman $\mathrm{AB}$. Kidney and pancreas transplantation in the United States, 1998-2007: Access for patients with diabetes and end-stage renal disease. Am J Transplant 2009; 9: 894906.

5. Dudley CR, Johnson RJ, Thomas HL, Ravanan R, Ansell D. Factors that influence access to the national renal transplant waiting list. Transplantation 2009; 88: 96-102. 
6. Bloembergen WE, Mauger EA, Wolfe RA, Port FK. Association of gender and access to cadaveric renal transplantation. Am J Kidney Dis 1997; 30: 733-738.

7. Ojo A, Port FK. Influence of race and gender on related donor renal transplantation rates. Am J Kidney Dis 1993; 22: 835-841.

8. Sequist TD, Narva AS, Stiles SK, Karp SK, Cass A, Ayanian JZ. Access to renal transplantation among American Indians and Hispanics. Am J Kidney Dis 2004; 44: 344-352.

9. Ayanian JZ, Cleary PD, Weissman JS, Epstein AM. The effect of patients' preferences on racial differences in access to renal transplantation. N Engl J Med 1999; 341: 1661-1669.

10. Navaneethan SD, Singh S. A systematic review of barriers in access to renal transplantation among African Americans in the United States. Clin Transplant 2006; 20: 769-775.

11. Tonelli M, Klarenbach S, Rose C, Wiebe N, Gill J. Access to kidney transplantation among remote- and rural-dwelling patients with kidney failure in the United States. JAMA 2009; 301: 1681-1690.

12. Axelrod DA, Guidinger MK, Finlayson $S$ et al. Rates of solid-organ wait-listing, transplantation, and survival among residents of rural and urban areas. JAMA 2008; 299: 202-207.

13. Garg PP, Frick KD, Diener-West M, Powe NR. Effect of the ownership of dialysis facilities on patients' survival and referral for transplantation. N Engl J Med 1999; 341: 1653-1660.

14. Chapman J, Russ G. Geographic variance in access to renal transplantation in Australia. Transplantation 2003; 76: 1403-1406.

15. Miranda B, Canon J, Cuende N, Garrido G, Naya MT, FernandezZincke E. Disparities in access to liver transplantation in Spain. Transplantation 2003; 76: 1398-1403.

16. Rudge CJ, Fuggle SV, Burbidge KM. Geographic disparities in access to organ transplantation in the United Kingdom. Transplantation 2003; 76: 1395-1398.

17. Ashby VB, Kalbfleisch JD, Wolfe RA, Lin MJ, Port FK, Leichtman $A B$. Geographic variability in access to primary kidney transplantation in the United States, 1996-2005. Am J Transplant 2007; 7: 1412-1423.

18. Tuttle-Newhall JE, Krishnan SM, Levy MF, McBride V, Orlowski JP, Sung RS. Organ donation and utilization in the United States: 1998-2007. Am J Transplant 2009; 9: 879-893.

19. U.S. Census Bureau, Population Division, County-Characteristics. Annual Estimates of the Resident Population by Sex, Race Alone, and Hispanic Origin for Counties: April 1, 2000 to July 1, 2008. 2008 August 14, 2009; Available from: http://www.census.gov/ popest/counties/asrh/CC-EST2008-RACE6.html

20. Social Security Administration Death Master File. Federal Computer Products Center, National Technical Information Service, U.S. Department of Commerce, Springfield, VA; In., 2009.

21. Scientific Registry of Transplant Recipients. 2009; Available from: http://www.ustransplant.org

22. Ladin K, Rodrigue JR, Hanto DW. Framing disparities along the continuum of care from chronic kidney disease to transplantation: Barriers and interventions. Am J Transplant 2009; 9: 669-674.

23. Organ Procurement and Transplantation Network. Health Resources and Services Administration, HHS. Final rule. Fed Regist 1999;64:56650-56661.

24. Kemmer N, Safdar K, Kaiser T, Zacharias V, Neff GW. Impact of geographic location on access to liver transplantation among ethnic minorities. Transplantation 2008; 85: 166-170.

25. Roberts JP, Dykstra DM, Goodrich NP, Rush SH, Merion RM, Port FK. Geographic differences in event rates by model for end-stage liver disease score. Am J Transplant 2006; 6: 2470-2475.
26. Ellison MD, Edwards LB, Edwards EB, Barker CF. Geographic differences in access to transplantation in the United States. Transplantation 2003; 76: 1389-1394.

27. Roudot-Thoraval F, Romano P, Spaak F, Houssin D, Durand-Zaleski I. Geographic disparities in access to organ transplant in France. Transplantation 2003; 76: 1385-1388.

28. Reddan D, Klassen P, Frankenfield DL et al. National profile of practice patterns for hemodialysis vascular access in the United States. J Am Soc Nephrol 2002; 13: 2117-2124.

29. Schold JD, Harman JS, Chumbler NR, Duncan RP, Meier-Kriesche $\mathrm{HU}$. The pivotal impact of center characteristics on survival of candidates listed for deceased donor kidney transplantation. Med Care 2009; 47: 146-153.

30. Leichtman AB, Cohen $D$, Keith $D$ et al. Kidney and pancreas transplantation in the United States, 1997-2006: The HRSA Breakthrough Collaboratives and the 58 DSA Challenge. Am J Transplant 2008; 8: 946-957.

31. Berthoux FC. Transplantation: Tackling inactivity on the waiting list for transplantation. Nat Rev Nephrol 2009; 5: 308-309.

32. Delmonico FL, McBride MA. Analysis of the wait list and deaths among candidates waiting for a kidney transplant. Transplantation 2008; 86: 1678-1683.

33. Nguyen K, Ashby VB, Kalbfleisch JD, Krishnan SM, Rao P, Leichtman AB. Marked Increase in the use of inactive status on the kidney transplant waiting list. Am J Transplantat 2008; 8(Suppl 2): 198.

34. Alexander GC, Sehgal AR. Barriers to cadaveric renal transplantation among blacks, women, and the poor. JAMA 1998; 280: 1148-1152.

35. Alexander GC, Sehgal AR. Why hemodialysis patients fail to complete the transplantation process. Am J Kidney Dis 2001; 37: 321328.

36. Arthur T. The role of social networks: A novel hypothesis to explain the phenomenon of racial disparity in kidney transplantation. Am J Kidney Dis 2002; 40: 678-681.

37. Wolfe RA, Ashby VB, Milford EL et al. Differences in access to cadaveric renal transplantation in the United States. Am J Kidney Dis 2000; 36: 1025-1033.

38. Ayanian JZ, Cleary PD, Keogh JH, Noonan SJ, David-Kasdan JA, Epstein AM. Physicians' beliefs about racial differences in referral for renal transplantation. Am J Kidney Dis 2004; 43: 350357.

39. Clark CR, Hicks LS, Keogh JH, Epstein AM, Ayanian JZ. Promoting access to renal transplantation: The role of social support networks in completing pre-transplant evaluations. J Gen Intern Med 2008; 23: 1187-1193.

40. Epstein AM, Ayanian JZ, Keogh JH et al. Racial disparities in access to renal transplantation-clinically appropriate or due to underuse or overuse? N Engl J Med 2000; 343(21): 1537-1544.

41. Callender CO, Bey AS, Miles PV, Yeager CL. A national minority organ/tissue transplant education program: The first step in the evolution of a national minority strategy and minority transplant equity in the USA. Transplant Proc 1995; 27: 14411443.

42. Callender CO, Miles PV. Institutionalized racism and end-stage renal disease: Is its impact real or illusionary? Semin Dial 2004; 17: 177-180.

43. Callender CO, Miles PV, Hall MB. National MOTTEP: Educating to prevent the need for transplantation. Minority Organ Tissue Transplant Education Program. Ethn Dis 2002; 12: S1, 34-37. 